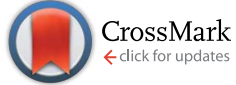

Cite this: J. Mater. Chem. B, 2014, 2 , 6283

Received 18th April 2014

Accepted 9th July 2014

DOI: $10.1039 / c 4 t b 00619 d$

www.rsc.org/MaterialsB

\section{Silver- and sulfadiazine-loaded nanostructured silica materials as potential replacement of silver sulfadiazine}

\author{
Ágnes Szegedi, ${ }^{* a}$ Margarita Popova, ${ }^{\mathrm{b}}$ Krassimira Yoncheva, ${ }^{\mathrm{c}}$ Judit Makk, ${ }^{\mathrm{d}}$ \\ Judith Mihály ${ }^{a}$ and Pavletta Shestakova ${ }^{b}$
}

Silver sulfadiazine (AgSD) is the leading topical antibacterial agent for the treatment of burn wound infections. Antibacterial effect of AgSD is limited by its poor aqueous solubility, and antibacterial activity develops only by decomposition of AgSD to silver ions and sulfadiazine. In this study, it is for the first time that application of silver-modified nanoporous silica carriers (MCM-41 or SBA-15) loaded with sulfadiazine (SD), instead of silver sulfadiazine, overcoming the abovementioned disadvantages has been demonstrated. By direct or post synthesis methods, 5-15 nm sized silver nanoparticles can be stabilized in the channels or on the outer surface of nanoporous silica supports; moreover, the empty channels can be loaded by SD molecules. The SD-loaded, silver-modified materials show sustained release properties and similar or even better antimicrobial properties than AgSD. Adsorption of AgSD on nanoporous silica particles significantly improves its water solubility.

\section{Introduction}

Silver sulfadiazine (AgSD) is an effective and widely used antimicrobial agent to avoid bacterial infections and external contamination., ${ }^{\mathbf{1} 2}$ Topical antibiotic therapy is essential for treatment of burns. Silver sulfadiazine is the leading topical agent to control bacterial infections in second-degree burn wounds. AgSD binds to cell components including DNA and causes membrane damage. AgSD is the silver salt of sulfadiazine, a polymeric type molecule, in which silver ion is tetracoordinated and surrounded by three different deprotonated sulfadiazine molecules, whereas each sulfadiazine molecule is bound to three different silver ions. ${ }^{3,4}$ The polymeric nature of AgSD contributes also to its very low solubility in aqueous systems $\left(3.4 \mathrm{mg} \mathrm{L}^{-1}\right.$ at $\left.\mathrm{pH}=6.8\right) .{ }^{5}$ Insolubility can result in minimum toxic potential to microorganisms, and in difficulties to incorporate it to polymeric/inorganic supports. However, according to Fox, antibacterial activity develops only by decomposition of AgSD to silver ions and sulfadiazine. ${ }^{6,7}$ The silver ion slowly dissociated from AgSD is the antimicrobially active part of the compound, whereas sulfadiazine has a supportive effect, but its concentration can be subinhibitory. Therefore, nowadays,

\footnotetext{
${ }^{a}$ Research Centre for Natural Sciences, Institute of Materials and Environmental Chemistry, Hungarian Academy of Sciences, Magyar tudósok körútja 2., 1117 Budapest, Hungary. E-mail: szegedi.agnes@ttk.mta.hu

${ }^{b}$ Institute of Organic Chemistry with Centre of Phytochemistry, Bulgarian Academy of Sciences, 1113 Sofia, Bulgaria

'Faculty of Pharmacy, 2 Dunav Str., 1000 Sofia, Bulgaria

${ }^{d}$ Eötvös Loránd University, Department of Microbiology, Budapest, Hungary
}

research is focusing on the enhancement of the solubility and the antibacterial efficacy by formulating AgSD as nanoparticles, ${ }^{8}$ nanosuspensions ${ }^{9}$ or by its incorporation to different types of natural or synthetic polymer, lipid- or dendrimer-based drug delivery platforms. ${ }^{\mathbf{1 0 - 1 4}}$ Moreover, biocompatible polymer wound dressing materials (semipermeable films, foams, hydrogels, hydrocolloids and xerogels) are intensively investigated in order to accelerate wound healing by preventing both fluid loss and bacterial infection by controlled antibacterial release..$^{15-20}$

In recent years, nanoporous silica-based drug delivery systems have also been developed. ${ }^{21,22}$ Nanoporous silica carriers (SBA-15 or MCM-41) are biocompatible materials, and they have the capability to load nanosized metal particles into the channels and can be functionalized with organic groups. ${ }^{23,24}$ The functionalization of the silica surface (inside the channels and/or the outer surface) with organic groups not only can enhance the adsorption of drug molecules but facilitates the interaction with biopolymers in order to produce organicinorganic composite materials with tailor made drug delivery properties. Thus, the effect of controlled drug release can be combined with the antibacterial effect of metallic nanoparticles such as silver. Silver nanoparticles can be stabilized in the channels or on the outer surface of mesoporous silica supports; moreover, the empty channels can be loaded by sulfadiazine molecules. With this bottom-up approach, a drug delivery system possessing the advantages of AgSD with improved bioavailability and antibacterial effect can be designed. Silver nanoparticle containing nanoporous silica materials can be easily synthesized by direct synthesis or by post-synthesis modification (template ion-exchange) methods. ${ }^{25,26}$ 
In this study, silver nanoparticle containing mesoporous silica Ag-SBA-15 and Ag-MCM-41 carriers were loaded with sulfadiazine in order to prepare a drug delivery system, which overcomes the disadvantages of AgSD and possesses improved bioavailability and solubility. To the best of our knowledge, this is the first attempt to substitute AgSD with silver- and sulfadiazine-loaded nanoporous silica materials, possessing high antibacterial activity.

\section{Experimental section}

\subsection{Synthesis of Si-MCM-41 and Ag-MCM-41 materials}

Spherical nanosized (100 nm \pm 50) MCM-41 particles were prepared according to the procedure of Huh et al. ${ }^{27}$ This sol-gel procedure is carried out at $80{ }^{\circ} \mathrm{C}$ in water solution with $\mathrm{NaOH}$ as a catalyst. The silica source was tetraethyl orthosilicate (TEOS), and hexadecyltrimethyl-ammonium bromide $\left(\mathrm{C}_{16} \mathrm{TMABr}\right)$ was applied as template. The relative molar composition of the reaction mixture was as follows: 1TEOS : $0.12 \mathrm{C}_{16} \mathrm{TMABr}: 0.31 \mathrm{NaOH}: 1190 \mathrm{H}_{2} \mathrm{O}$. The formed gel was aged at $80{ }^{\circ} \mathrm{C}$ for $2 \mathrm{~h}$, then washed with distilled water until neutral $\mathrm{pH}$, and dried at room temperature. Silver nanoparticles were loaded to the silica carrier by template ion-exchange method (TIE) suggested by Iwamoto et al. and Gac et al. ${ }^{\mathbf{2 6 , 2 8}}$ According to Iwamoto et al.,$^{28}$ by TIE method, the different types of transition metals can be incorporated into the structure of MCM-41 in different amounts (1.5-7 wt\%, Si/Me 100-20), depending on the chemical nature of the metal. In the case of silver $5.5 \mathrm{wt} \%$ final silver content could be achieved with $\mathrm{Si} / \mathrm{Ag}=5$ ratio in ion-exchange solution. The procedure is as follows: the template containing MCM-41 material was ion-exchanged by refluxing it at $80{ }^{\circ} \mathrm{C}$ with $0.036 \mathrm{M} \mathrm{AgNO}_{3}$ solution $\left(50 \mathrm{~mL} \mathrm{~g}^{-1}\right.$ MCM-41) for $20 \mathrm{~h}$, then filtered on $0.2 \mu \mathrm{m}$ membrane filter, and finally washed with distilled water to make it chloride-free. The ion-exchanged material was heat treated in air at $550{ }^{\circ} \mathrm{C}$ for $5 \mathrm{~h}$ with a heating rate of $1{ }^{\circ} \mathrm{C} \mathrm{min}^{-1}$. Silver containing MCM-41 sample prepared by template ion-exchange method was designated as Ag-MCM-41.

\subsection{Synthesis of Si-SBA-15 and Ag-SBA-15 supports}

Pure silica SBA-15 was synthesized according to the original procedure of Zhao et al. $^{29}$ applying Pluronic 123 triblock copolymer (BASF, $\mathrm{P} 123, \mathrm{EO}_{20} \mathrm{PO}_{70} \mathrm{EO}_{20}, M_{\mathrm{w}}=5800$ ) as a template and TEOS as a silica source in the acidic media of $2 \mathrm{~N} \mathrm{HCl}$.

Mesoporous Ag-SBA-15 material was synthesized by direct hydrothermal synthesis also in the presence of P123 as a template and TEOS as a silica source by modifying the procedure of Zhu et al. ${ }^{25}$ However, to avoid the precipitation of $\mathrm{AgCl}, \mathrm{HNO}_{3}$ was applied as acidic media. In a typical synthesis procedure, $4 \mathrm{~g}$ of P123, $120 \mathrm{~g}$ of deionized water and $18.8 \mathrm{~g}$ of $65 \mathrm{wt} \%$ nitric acid were mixed and stirred for $1 \mathrm{~h}$ at $40{ }^{\circ} \mathrm{C} .0 .34 \mathrm{~g}$ of silver nitrate $(\mathrm{Si} / \mathrm{Ag}=20)$ was added to the solution and stirred for $1 \mathrm{~h}$ at the same temperature in the dark. Then, $8.4 \mathrm{~g}$ of TEOS was slowly added to the solution and stirred for $20 \mathrm{~h}$ at $40{ }^{\circ} \mathrm{C}$ in the dark. The final molar ratio of the synthesis mixture was as follows: 1TEOS : $0.017 \mathrm{P} 123: 0.05 \mathrm{AgNO}_{3}: 4.93 \mathrm{HNO}_{3}: 177.9 \mathrm{H}_{2} \mathrm{O}$.
The precipitated white suspension was aged at $100{ }^{\circ} \mathrm{C}$ for $48 \mathrm{~h}$ in a Teflon-lined stainless steel autoclave without stirring. The product was filtered and washed with deionized water until it was nitrate-free. The template was removed by calcination in air at $450{ }^{\circ} \mathrm{C}$ for $5 \mathrm{~h}$ with a heating rate of $1{ }^{\circ} \mathrm{C} \mathrm{min}{ }^{-1}$. Silver containing SBA-15 sample prepared by a direct, one-pot synthesis method was designated as Ag-SBA-15.

\subsection{Loading of sulfadiazine and silver sulfadiazine on silver modified and non-modified nanoporous materials}

Sulfadiazine (SD) and AgSD loading was carried out in a mixture of acetone and methanol $(1: 1)$ at $37{ }^{\circ} \mathrm{C}$ by applying $250 \mathrm{mg}$ of SD or AgSD per $250 \mathrm{mg}$ support. $250 \mathrm{mg}$ SD was dissolved in 120 $\mathrm{mL}$ of solvent mixture, whereas AgSD in $250 \mathrm{~mL}$. After incubation for $24 \mathrm{~h}$, the mixtures were centrifuged at $15000 \mathrm{rpm}$, rinsed with distilled water, separated by a second centrifugation, and finally dried at room temperature under vacuum. SDloaded Ag-MCM-41 and Ag-SBA-15 samples were designated as Ag-MCM-41/SD and Ag-SBA-15/SD, respectively. AgSD-loaded samples were designated as MCM-41/AgSD and SBA-15/AgSD.

\subsection{Characterization}

X-ray diffraction patterns were recorded by a Philips PW 1810/ 3710 diffractometer with Bragg-Brentano parafocusing geometry by applying monochromatized $\operatorname{CuK} \alpha(\lambda=0.15418 \mathrm{~nm})$ radiation $(40 \mathrm{kV}, 35 \mathrm{~mA})$ and a proportional counter. Metallic silver, sulfadiazine and silver sulfadiazine were identified based on JCPDS ICDD database or on the XRD pattern of the parent commercial drug. The corresponding ICDD card numbers were: Ag4f 04-0783, SD 39-1841, and AgSD 37-1555. Crystallite size of metallic silver was determined by the Scherrer equation evaluating the FWMH values with full profile fitting method.

Nitrogen physisorption measurements were carried out at 77 K using Quantachrome Autosorb 1C apparatus. The specific surface area was calculated by the BET method in the range of relative pressures from 0.02 to 0.1 . The pore-size distribution was calculated from desorption branch of the isotherms with the $\mathrm{BJH}$ method. Silica samples were pre-treated at $350{ }^{\circ} \mathrm{C}$, whereas drug loaded materials at $80{ }^{\circ} \mathrm{C}$ for $5 \mathrm{~h}$ before measurements.

Silver content of the prepared samples was determined by atomic absorption spectroscopy (AAS) by applying $\mathrm{AgNO}_{3}$ standard solution after digesting the silver containing samples in $\mathrm{HF}$ and $\mathrm{HNO}_{3}$.

TEM images were obtained by using a MORGAGNI 268D TEM (100 kV; W filament; point-resolution $=0.5 \mathrm{~nm}$ ) electron microscope. Samples were suspended in small amount of ethanol and a drop of suspension was deposited onto copper grid covered by carbon supporting film and dried at room temperature.

Thermogravimetric measurements were performed with a Setaram TG92 instrument with a heating rate of $5{ }^{\circ} \mathrm{C} \mathrm{min}^{-1}$ in nitrogen flow.

Attenuated total reflection infrared (ATR-FTIR) spectra were recorded by means of a Varian Scimitar 2000 FTIR spectrometer equipped with a MCT (mercury cadmium-telluride) detector 
and a single reflection ATR unit (SPECAC "Golden Gate") with diamond ATR element. In general, 128 scans and $4 \mathrm{~cm}^{-1}$ resolution were applied. For all spectra ATR-correction was performed (Varian ResPro 4.0 software).

MAS NMR spectra were recorded on a Bruker Avance II +600 NMR spectrometer operating at $600.13 \mathrm{MHz}$ proton frequency $\left(150.90 \mathrm{MHz}\right.$ for ${ }^{13} \mathrm{C}$ ) using $4 \mathrm{~mm}$ solid state $\mathrm{CP} / \mathrm{MAS}$ dual probe head. The samples were loaded in $4 \mathrm{~mm}$ zirconia rotors and spun at magic angle spinning (MAS) rate of $6 \mathrm{kHz}$ in all experiments. ${ }^{13} \mathrm{C}$ NMR spectra were acquired with $8 \mathrm{~K}$ time domain data points, spectrum width of $50 \mathrm{kHz}, 256$ scans and a recycle delay of $5 \mathrm{~s}$ using a cross polarization pulse sequence with total suppression of side bands (cptoss) from Bruker Topspin library. The spectra were processed with an exponential window function (line broadening factor 10) and zero filled to $16 \mathrm{~K}$ data points.

\subsection{In vitro release studies}

For in vitro release studies, $10 \mathrm{mg}$ of the drug-loaded silica particles were incubated in $200 \mathrm{~mL}$ phosphate buffer $(\mathrm{pH}=5.5)$ at $37^{\circ} \mathrm{C}$ under stirring $(100 \mathrm{rpm})$. At appropriate time intervals, $5 \mathrm{~mL}$ samples were withdrawn and replaced by fresh buffer. The withdrawn samples were centrifuged at $15000 \mathrm{rpm}$ for $15 \mathrm{~min}$ and the concentration of the released drug was determined by UV-spectrophotometry at a wavelength of $262 \mathrm{~nm}$ (Hewlett Packard 8452A). ${ }^{30,13}$

\subsection{In vitro antibacterial tests}

Antibacterial activity of silver and SD containing samples was measured on agar plates inoculated with Pseudomonas aeruginosa (ATCC 9027), Escherichia coli (ATCC 8739), Staphylococcus aureus (ATCC 6538), and Streptococcus pyogenes (ATCC 19615) strains using the agar diffusion test.

The fresh bacterial suspensions were composed of frozen bacterial cultures. E. coli, S. aureus and P. aeruginosa were grown in tryptone soya broth (TSB, DSMZ 545) at $37.0 \pm 1.0{ }^{\circ} \mathrm{C}$ for $24 \mathrm{~h}$. Water suspension of $S$. pyogenes was prepared of freshly isolated colonies on PPLO agar (beef heart infusion agar, Difco) after incubation at $37.0 \pm 1.0{ }^{\circ} \mathrm{C}$ for $24 \mathrm{~h}$. Before agar plates were inoculated with bacterial strains the BIOLOG Gram positive blue standard was used to match the turbidity of bacterial culture suspensions $\left(\sim 10^{6}\right.$ colony forming units per $\mathrm{mL}$ $\left.\left[\mathrm{CFU} \mathrm{mL} \mathrm{mL}^{-1}\right]\right) .100 \mu \mathrm{L}$ of the prepared bacterial suspension $\left(\sim 10^{6} \mathrm{CFU} \mathrm{mL}{ }^{-1}\right)$ was spread on Petri dish containing nutrient agar medium (DSMZ 1), and on PPLO agar medium for $S$. pyogenes, respectively.

Two methods were applied to compare the antibacterial properties of the studied samples. By the first method, $12 \mathrm{~mm}$ diameter discs (weight about 15-20 mg) were pressed aseptically on the silver- and SD-containing samples, and they were gently placed on the surface of the wet agar plates. By the second, well diffusion method two cavities were made in the agar plates using a cork borer (10 $\mathrm{mm}$ diameter). The cavities were filled with $200 \mu \mathrm{L}$ sterilized distilled water suspension of the samples $\left(10 \mathrm{mg} \mathrm{mL}^{-1}\right)$.

The agar plates were incubated in a refrigerator $\left(4 \mathrm{~h}, \sim 9{ }^{\circ} \mathrm{C}\right.$, promoting diffusion of antibacterial samples and inhibiting bacterial growth) before being placed in a thermostat (24 and 48 hours at $37.0 \pm 1.0^{\circ} \mathrm{C}$ ). After incubation, the antimicrobial efficacy of each test material was determined by measuring the diameter of the clear zone, i.e. the zone of inhibition around the antimicrobial materials in millimeters. Experiments were performed in triplicate and the average value was determined.

\section{Results and discussion}

\subsection{Characterization of silver-containing nanoporous silica materials}

Low- and high-angle XRD powder patterns of pure silica and silver containing SBA-15 and MCM-41 materials, and their SD
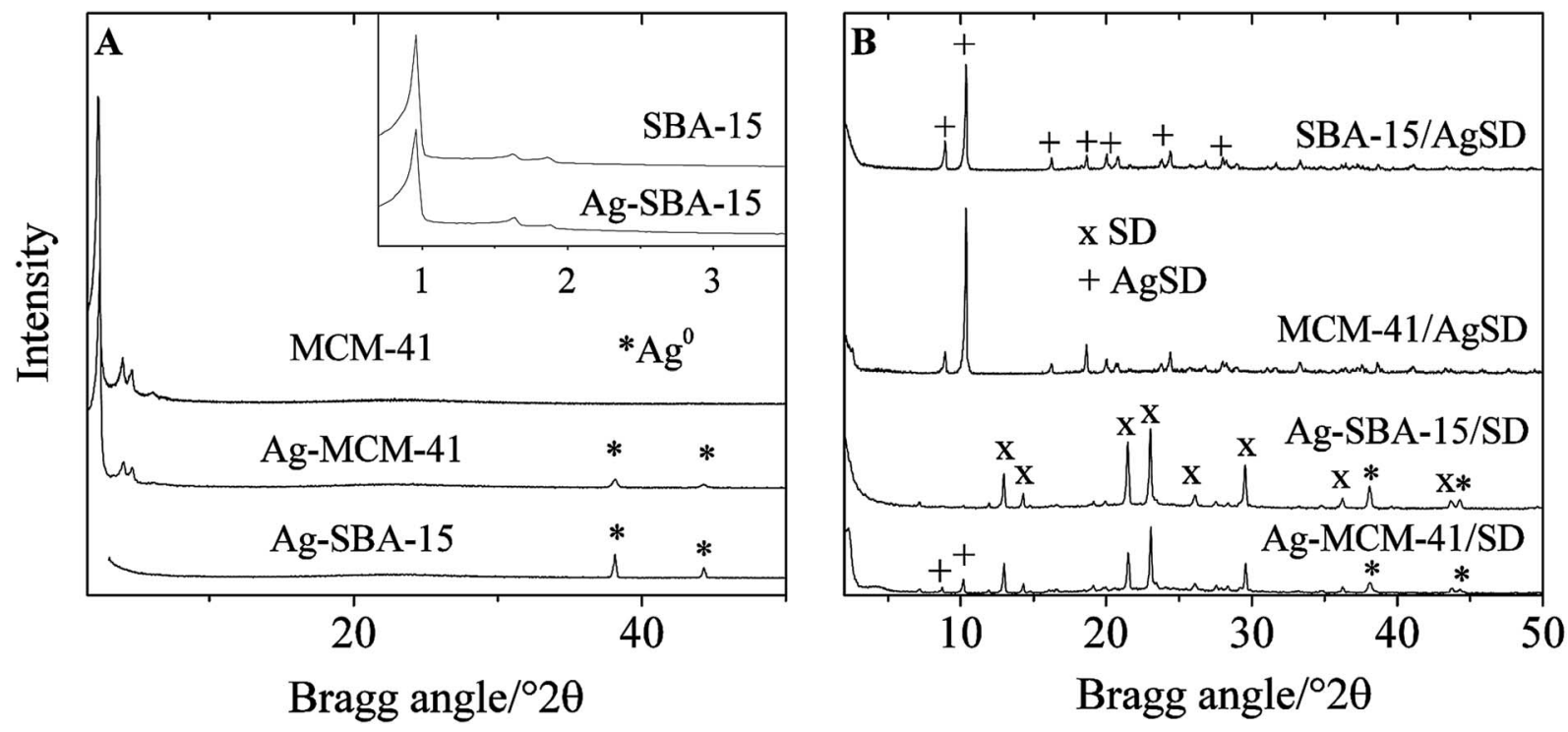

Fig. 1 XRD patterns of silver-containing nanoporous pure silica and silver-modified silica samples (A) and their SD-and AgSD-loaded varieties (B). 
Table 1 Composition and textural properties of the studied samples

\begin{tabular}{|c|c|c|c|c|c|c|}
\hline Samples & $\begin{array}{l}\text { Ag content }{ }^{a} \\
{\left[\mathrm{mmol} \mathrm{g}_{\text {calc }}{ }^{-1}\right]}\end{array}$ & $\begin{array}{l}\text { SD content } \\
{\left[\mathrm{mg} \mathrm{g}^{-1}\right]}\end{array}$ & $a_{0}^{b}[\mathrm{~nm}]$ & $\begin{array}{l}\text { BET surf. area } \\
{\left[\mathrm{m}^{2} \mathrm{~g}^{-1}\right]}\end{array}$ & $\begin{array}{l}\text { Total pore vol. } \\
{\left[\mathrm{cm}^{3} \mathrm{~g}^{-1}\right]}\end{array}$ & $\mathrm{PD}^{c}[\mathrm{~nm}]$ \\
\hline MCM-41 & - & - & 4.4 & 1175 & 0.97 & 2.7 \\
\hline Ag-MCM-41 & 0.52 & - & 4.3 & 927 & 0.76 & 2.6 \\
\hline Ag-SBA-15 & 0.66 & - & 10.6 & 698 & 1.08 & 6.6 \\
\hline Ag-MCM-41/SD & 0.52 & 305 & 4.3 & 233 & 0.18 & 2.3 \\
\hline SBA-15/AgSD & 1.22 & 307 & 10.2 & 316 & 0.40 & 4.1 \\
\hline
\end{tabular}

${ }^{a}$ Determined by AAS method after digestion in $\mathrm{HF}$ and $\mathrm{HNO}_{3} \cdot{ }^{b}$ Unit cell parameter $\left(a_{0}=2 d_{10} 0(3)^{-1 / 2}\right) .{ }^{c}$ Mean pore diameter calculated by the BJH method.

and AgSD loaded varieties are shown in Fig. 1. XRD pattern of Ag-SBA-15 sample at low $2 \theta^{\circ}$ region show the formation of typical, well-ordered $2 \mathrm{D}$ hexagonal $(p 6 \mathrm{~mm})$ structure (Fig. 1 inset) with the intense (100) reflection and the appearance of the higher indexed (110) and (200) peaks. Compared to a pure silica SBA-15 some intensity decrease can be observed. XRD patterns at higher angles show the presence of a separate metallic silver phase. Ag-MCM-41 samples prepared by template ion-exchange method also show the typical patterns of highly ordered hexagonal phase. The intensity of (100) reflection is lower than that of parent silica variety. Reflections of metallic silver can also be observed at higher angles, but their intensity is smaller than on Ag-SBA-15 sample. According to AAS results (Table 1), the silver content of Ag-MCM-41 sample is slightly lower than that of Ag-SBA-15. The appearance of metallic silver on the pattern is an indication of the formation of silver crystallites bigger than $\sim 5 \mathrm{~nm}$, according to the Scherrer equation. ${ }^{31,32}$ These nanoparticles can be found as a separate phase among the silica particles or attached to the outer surface of the silica support.

By comparing the intensity of $\mathrm{Ag}^{0}$ reflections of the prepared samples with that of a mechanical mixture of pure silica and metallic silver (not shown), it roughly corresponds to the

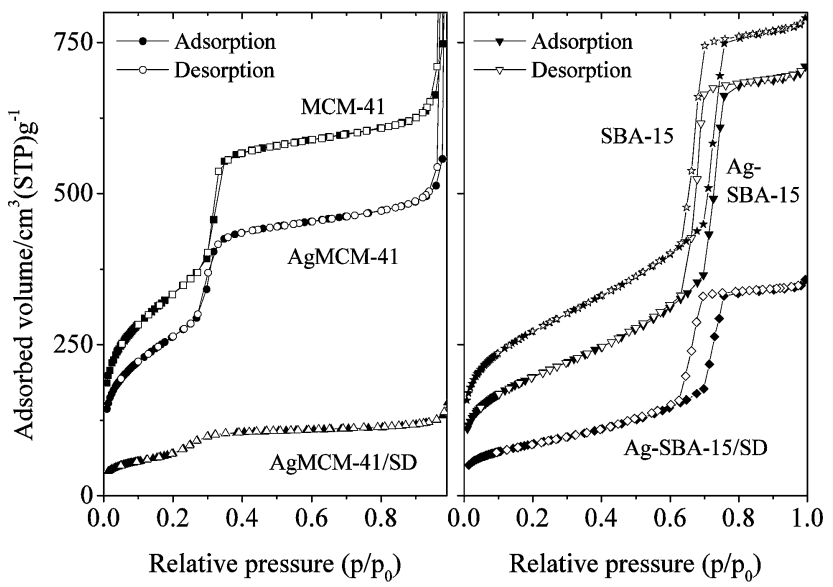

Fig. $2 \mathrm{~N}_{2}$ physisorption isotherms of pure silica, silver-containing and sulfadiazine-loaded varieties of nanoporous silica materials. expected value by the metal content of the materials (about 7 and $5.5 \mathrm{wt} \%$ for Ag-SBA-15 and Ag-MCM-41 samples, respectively). However, confinement of silver nanoparticles inside the channels of silica host, and also the incorporation of silver ions into the silica wall in terminal position of the silanol groups as $\mathrm{Ag}-\mathrm{O}-\mathrm{Si} \equiv \mathrm{O}_{3}$ species cannot be excluded. In contrast to other two and trivalent transition metals ( $\mathrm{Cu}, \mathrm{Ni}, \mathrm{Co}, \mathrm{Fe})$, monovalent metals cannot strongly interact with the silica structure during the heat treatment in the template removal step. ${ }^{33}$ They can be reduced by the decomposition products of template molecules and the formed small metallic particles can migrate to form bigger silver particles. Formation of ionic silver species connected to the silica framework is more probable in Ag-SBA-15 sample due to the more homogeneous distribution of silver ions by the sol-gel synthesis method and to the thicker walls of SBA15 structure.

No significant changes, only a small decrease can be observed in the unit cell and pore size of Ag-MCM-41 sample (Table 1) compared to the pure silica variety. This can be due to the deterioration effect of ion-exchange procedure. Heating the sample in water solution at $80{ }^{\circ} \mathrm{C}$ for $20 \mathrm{~h}$ can result in dissolution of silica and the decrease of ordering of honeycomb-like structure. Incorporation of $\mathrm{Ag}$ into the structure of SBA-15 is associated with more significant changes. The unit cell size and the pore size is increased, which can be either due to the isomorphous substitution of silicon atoms by the bigger silver ions or due to the change of synthesis medium from $\mathrm{HCl}$ to $\mathrm{HNO}_{3}$.

The above observations were supported also by the $\mathrm{N}_{2}$ adsorption data (Fig. 2). Textural parameters are summarized in Table 1. The nitrogen physisorption isotherms of pure silica and silver-modified MCM-41 materials show the typical IV type physisorption isotherm with a capillary condensation step of nitrogen between relative pressures of 0.2 and 0.3 . Compared to parent silica material the specific surface area and the total pore volume of Ag-MCM-41 sample are slightly lower, supporting the structure deterioration idea. The $\mathrm{N}_{2}$ adsorption isotherms of AgSBA-15 sample belong to the IV type with a H1 type hysteresis loop, typical for SBA-15 structures. The decrease of specific surface area and pore volume of Ag-SBA-15 sample compared to 
the silica one is minor and probably can be due to some pore blocking effect of small silver nanoparticles.

The modification by silver does not influence the original morphology of MCM-41 and SBA-15 materials as evidenced by TEM investigations (Fig. 3). Ag-MCM-41 shows the typical 100 $\mathrm{nm}$ spherical particles and the channel system is well preserved. Silver nanoparticles with different dispersities between 5 and 20 $\mathrm{nm}$ can be observed on the images. These results are in accordance with XRD results showing the presence of separate silver phase on the outer side of the particles. TEM images of AgSBA-15 sample exhibits a similar picture. 5-20 nm sized silver nanoparticles on the external surface, and smaller $5 \mathrm{~nm}$ particles inside the channels can be observed.

From the antibacterial point of view, the release of $\mathrm{Ag}^{+}$ions from the antibacterial material is essential. ${ }^{34}$ Silver ions can be released either from silver nanoclusters by their oxidation with

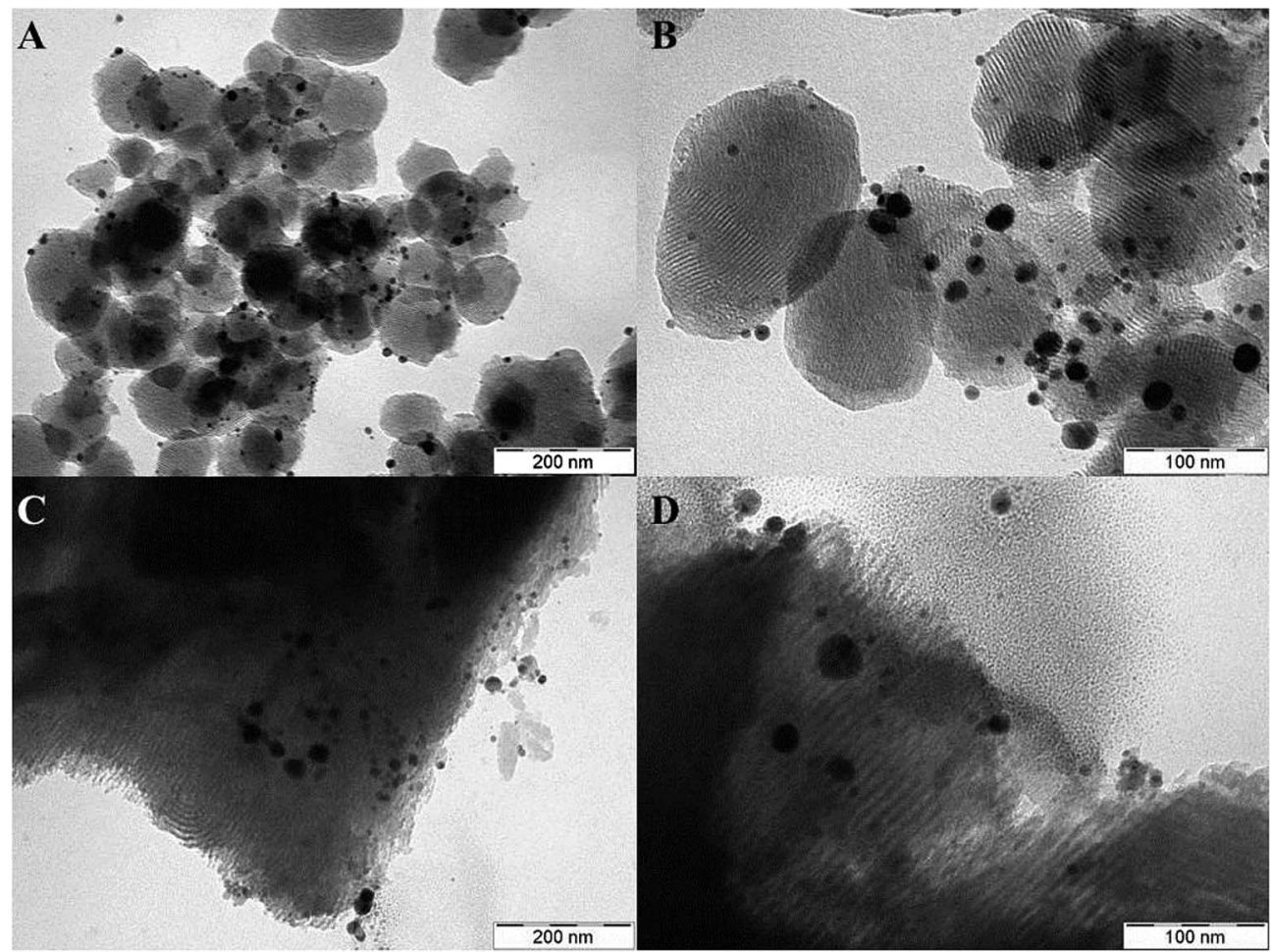

Fig. 3 TEM images of Ag-MCM-41 (A and B) and Ag-SBA-15 (C and D) samples.
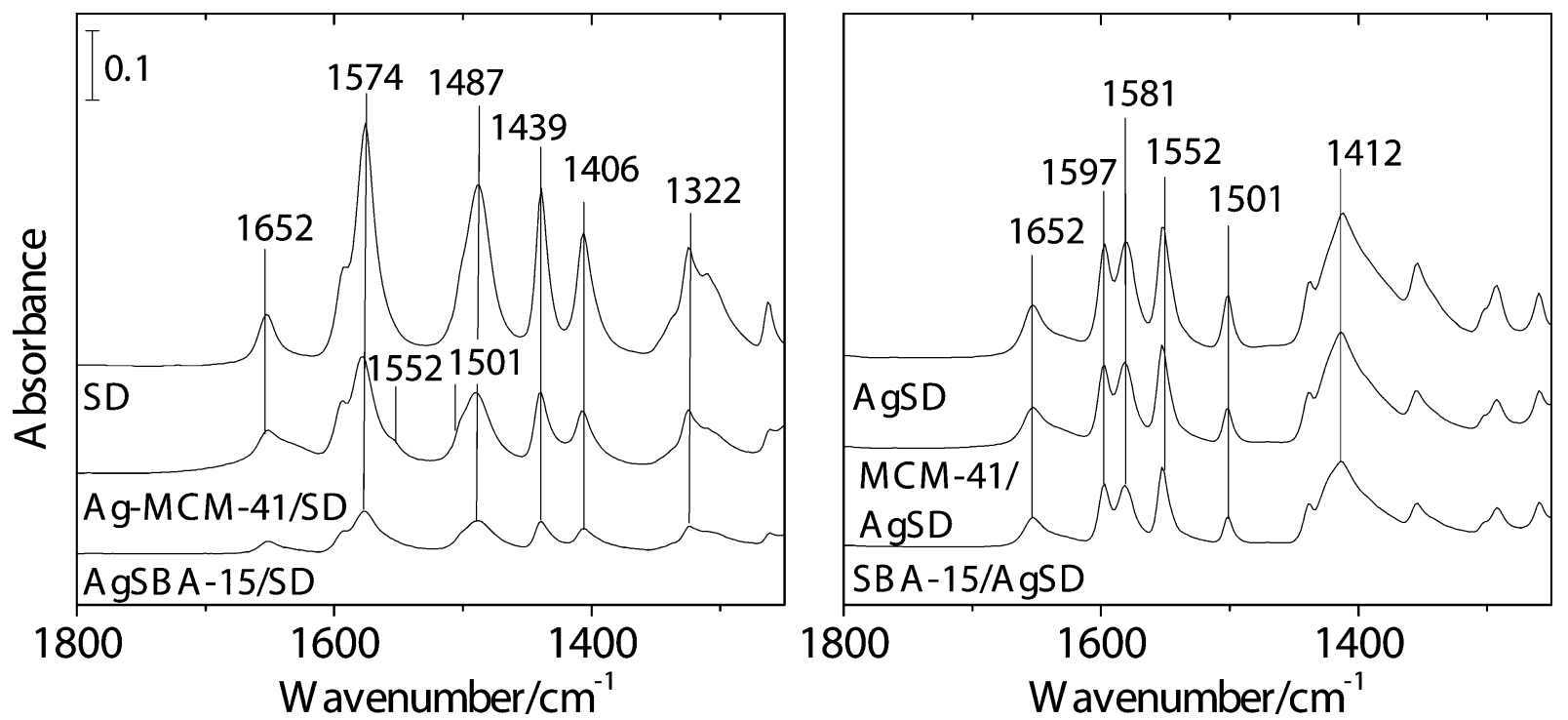

Fig. 4 FT-IR spectra of SD-loaded Ag-silica materials and AgSD loaded nanoporous silica samples. 
<smiles>Nc1ccc(S(=O)(=O)Nc2ncccn2)cc1</smiles><smiles>Nc1ccc(S(=O)(=O)Nc2ncccn2)cc1</smiles>

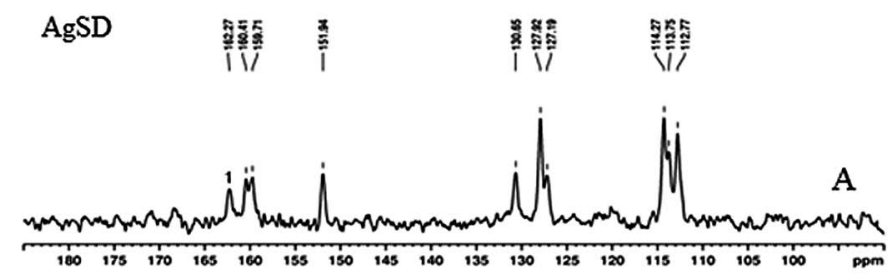

SD

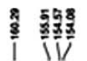

i
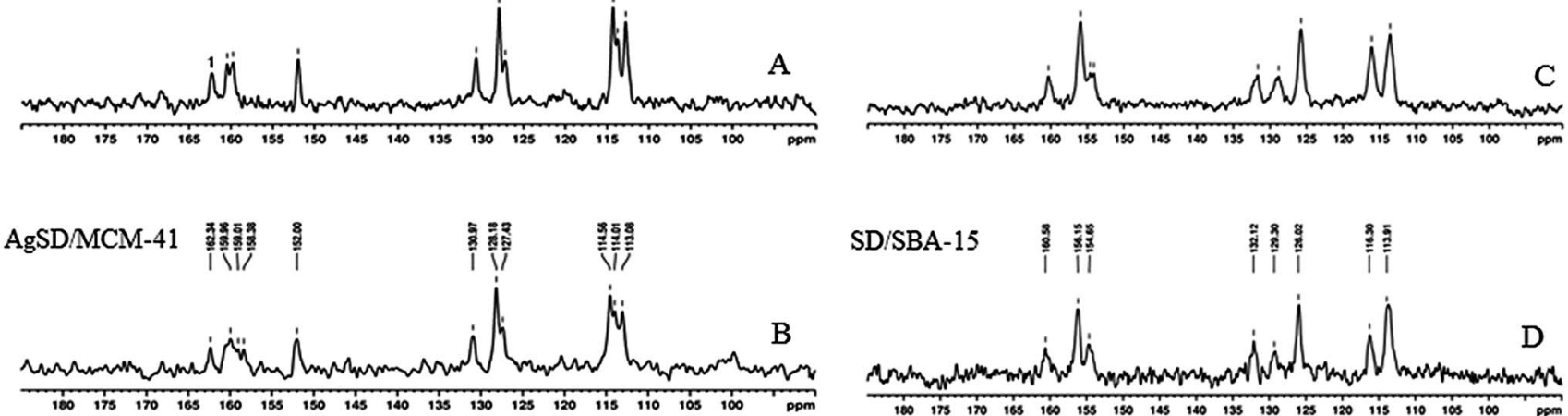

Fig. $5{ }^{13}$ C NMR spectra of SD-loaded Ag-SBA-15 and AgSD-loaded MCM-41 sample compared to pure SD and AgSD, respectively.

$\mathrm{H}_{3} \mathrm{O}^{+}$ions in water, ${ }^{30}$ or by the cleavage of $\mathrm{Ag}^{+}$ions connected to the silica wall through oxygen atoms. In this respect, solubility of the silica host also plays an important role. It is well known that due to their thinner walls and lower silica condensation rate, MCM-41-type silica materials show less stability towards water dissolution than SBA-15 ones. ${ }^{35}$ Therefore, higher amount of silver release is to be expected from silver-containing MCM41 materials. The silver ion dissolution capacity of the prepared samples was checked by stirring $0.2 \mathrm{~g}$ of Ag-SBA-15 and AgMCM-41 in $50 \mathrm{~mL}$ water for $24 \mathrm{~h}$ at $37 \pm 2{ }^{\circ} \mathrm{C}$. By AAS method, for Ag-MCM-41 sample, $4.7 \mathrm{mg} \mathrm{\textrm {Ag } ^ { + }} \mathrm{g}_{\text {calc }}{ }^{-1}$ was detected, whereas for Ag-SBA-15 sample, $2.9 \mathrm{mg} \mathrm{Ag}^{+} \mathrm{g}_{\text {calc }}{ }^{-1}$ was detected. These values correspond to $9 \%$ and $4.5 \%$ of the total silver content of the samples, respectively. Comparing these silver ion release values to other type of $\mathrm{Ag} / \mathrm{SiO}_{2}$ materials it represents average among the disperse values can be found in literature. For example, Kawashita et al. ${ }^{36}$ have found $0.2 \mathrm{mg} \mathrm{g}^{-1}$ silver ion release for silver-doped glasses, whereas Lu et al. ${ }^{37}$ determined $8.5 \mathrm{mg} \mathrm{g}^{-1}$ by silver nanoclusters encapsulated in porous silica nanospheres. These data support that in MCM-41 material the dissolution of silver is easier, and silver ions and metallic particles are not so strongly bound to the silica host due to the post-synthesis modification method. In SBA-15 support, because of the direct synthesis method, silver can be found embedded in the silica wall in the form of $\mathrm{Ag}-\mathrm{O}-\mathrm{Si} \equiv$ ionic species or confined inside the channels as small nanoparticles. These facts can explain the lower amount of released silver ions from Ag-SBA-15, but the higher silver content of the sample can compensate its lower release rate.

\subsection{Adsorption of sulfadiazine and silver sulfadiazine}

Sulfadiazine was adsorbed on silver-containing samples by dissolving SD in the mixture of methanol and acetone. SD penetrated into the channels of silica carrier and partial pore filling was achieved, evidenced by the $\mathrm{N}_{2}$ physisorption data (Fig. 2, Table 1). Correcting the pore volume data by the actual amount of silica carrier, $65-70 \%$ pore filling for MCM-41 samples and $10-30 \%$ loading can be calculated for SBA-15 support. According to the XRD patterns of the SD loaded silica materials (Fig. 1B), the remaining amount of SD can be found as

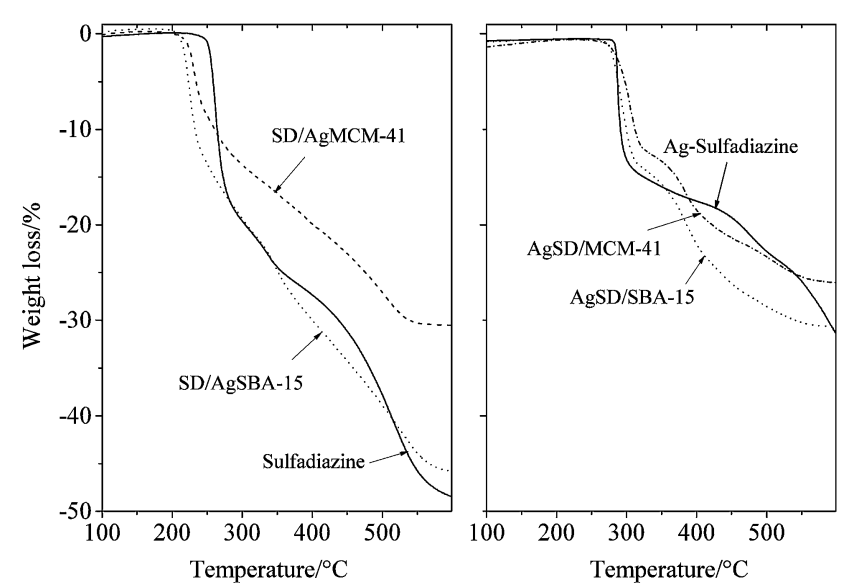

Fig. 6 TG curves of SD loaded Ag-MCM-41 and Ag-SBA-15 samples (A) and AgSD loaded pure silica MCM-41 and SBA-15 materials (B). 
a separate phase in the sample. Appearance of a small amount of AgSD can be also detected on Ag-MCM-41/SD sample. FT-IR investigations show that the loaded sulfadiazine does not react with the silica walls, i.e. mainly physical adsorption occurs. The surface silanol groups of silica are weak acids that cannot protonate the amino groups of sulfadiazine; therefore, no strong interaction between SD and silica matrix can be expected. As reported in our previous work ${ }^{24}$ surface functionalization of silica support by carboxylic groups can enhance the adsorption capacity and sustained release can be achieved by the electrostatically bonded sulfadiazine due to the formation of $\mathrm{COO}^{-} \mathrm{NH}_{3}^{+}$groups with the amino groups of sulfadiazine.

Ag-SBA-15/SD shows similar FT-IR spectral features like free SD (Fig. 4): $\mathrm{NH}_{2}$ bending of free amino groups $\left(\delta_{\mathrm{NH}_{2}}\right.$ at $\left.1652 \mathrm{~cm}^{-1}\right)$, phenyl skeletal vibrations $\left(\nu_{\mathrm{CC}}\right.$ at 1574 and $\delta_{\mathrm{CCH}}$ at $\left.1487 \mathrm{~cm}^{-1}\right)$ and pyrimidine skeletal vibrations $\left(\delta_{\mathrm{CCH}}\right.$ at 1439 and $\delta_{\mathrm{HCN}}$ at $\left.1406 \mathrm{~cm}^{-1}\right)$. The band at $1322 \mathrm{~cm}^{-1}$ belongs to the symmetrical stretching of $\mathrm{SO}_{2}$ groups. ${ }^{\mathbf{3 8 2 4}}$ However, in the spectrum of Ag-MCM-41/SD, small new bands (shoulders) at 1553 and $1501 \mathrm{~cm}^{-1}$ appear. These bands are to be found in the spectrum of AgSD, and they can be assigned to the shifted ring vibrations of pyrimidine due to the presence of $\mathrm{Ag}^{+}$ions. Appearance of the latter bands is an indication that a part of adsorbed SD is in the close vicinity of silver nanoparticles or silver ions remained connected to the silica walls inside the channels of Ag-MCM-41 after the template removal, in
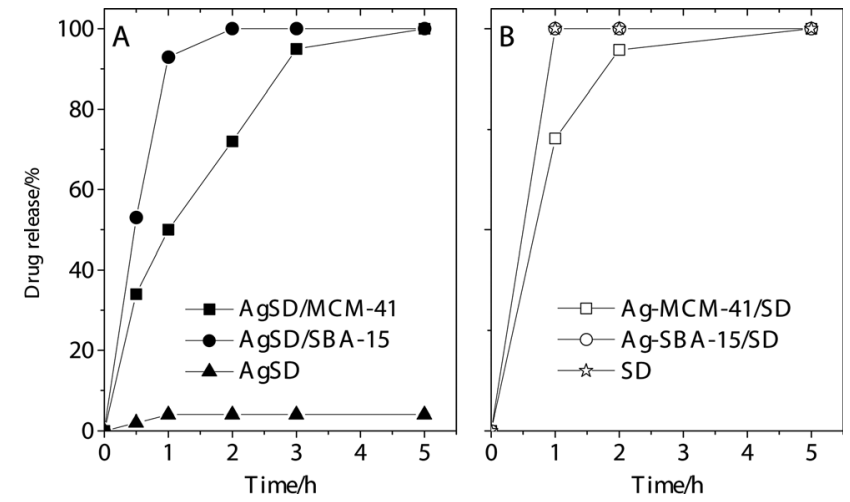

Fig. 7 In vitro release of SD from Ag-MCM-41 and Ag-SBA-15 carriers and AgSD release from MCM-41 and SBA-15 samples. accordance with XRD results. It should be noted that no spectral changes can be detected for adsorbed SD in non-modified pure silica MCM-41. ${ }^{24}$

AgSD was also adsorbed on pure silica materials to compare the physicochemical and antimicrobial properties with SDloaded, silver-modified varieties. $\mathrm{N}_{2}$ physisorption data (Table 1) show that despite its extremely low solubility, AgSD can penetrate into the channels of silica carriers and almost total pore filling in MCM-41 and partial pore filling for SBA-15 carrier can be detected. However, according to XRD patterns (Fig. 1B), the majority of AgSD can be found outside the channels. This is more pronounced for MCM-41 material, probably due to its lower pore volume and smaller pore size, hindering the penetration of the large polymeric molecule. $\mathrm{N}_{2}$ physisorption data indicate total pore filling; however, it might be that AgSD blocks only the entrances of pores.

Interaction of adsorbed AgSD with the silica carrier was investigated by FT-IR method. In AgSD, silver ion is coordinated to the deprotonated amine nitrogen, to the sulfonyl oxygen and to the pyrimidine nitrogen in the same chain. Each silver ion is coordinated to further pyrimidine nitrogen, forming a polymeric chain structure. ${ }^{39}$ The FT-IR spectrum (Fig. 4) of AgSD shows different features compared to SD. The phenyl skeletal vibrations are shifted towards higher wavenumber and the $\mathrm{C}-\mathrm{C}$ stretching vibrations of the aromatic ring are split (1597 and $1581 \mathrm{~cm}^{-1}$ ). Other characteristic AgSD vibrational bands can be found at 1552, 1501 and $1412 \mathrm{~cm}^{-1}$, which are assigned to pyrimidine skeletal vibrations. Spectra of AgSD-loaded MCM-41 and SBA-15 samples show similar spectral bands like free AgSD, indicating that $\mathrm{AgSD}$ is physisorbed on silica carriers.

Interaction of adsorbed AgSD with the silica carrier and of SD with Ag-containing mesoporous support was investigated by ${ }^{13} \mathrm{C}$ NMR. Fig. 5A and B show the ${ }^{13} \mathrm{C}$ spectra of pure AgSD and the AgSD loaded on MCM-41 material, respectively. The two spectra demonstrate similar spectral patterns; however, most of the signals of AgSD-loaded silica material are shifted downfield. These chemical shift changes are indicative for the new structural and chemical environment of AgSD molecules as a result of their incorporation into silica matrix. More significant differences between the ${ }^{13} \mathrm{C}$ spectra of the two samples are observed in the spectral region between 156 and $161 \mathrm{ppm}$, where the two peaks corresponding to $\mathrm{C} 2$ and $\mathrm{C} 4$ atoms from pyrimidine moiety are shifted upfield; moreover, a broad resonance partially overlapped with these signals appear in the

Table 2 Antibacterial activity of Ag-MCM-41, Ag-SBA-15 and SD loaded MCM-41 samples

\begin{tabular}{|c|c|c|c|c|c|c|}
\hline \multirow[b]{3}{*}{ Bacterial strains } & \multicolumn{6}{|c|}{ Zone of inhibition [mm] } \\
\hline & \multicolumn{2}{|c|}{ Ag-MCM-41 } & \multicolumn{2}{|c|}{ Ag-SBA-15 } & \multicolumn{2}{|c|}{ MCM-41/SD } \\
\hline & Disc & Susp. & Disc & Susp. & Disc & Susp. \\
\hline Escherichia coli ATCC 8739 & 1 & 5 & $1+1$ & 1 & $14^{a}$ & $14^{a}$ \\
\hline Staphylococcus aureus ATCC 6538 & 3 & 5.5 & 1 & 2 & 0 & 0 \\
\hline Pseudomonas aeruginosa ATCC 9027 & 2 & 2 & $1+2$ & 2 & 0 & 0 \\
\hline Streptococcus pyogenes ATCC 19615 & 3 & 7 & 3.5 & 6 & 0 & 0 \\
\hline
\end{tabular}

${ }^{a}$ Not totally clean inhibition zone. 
Table 3 Antibacterial activity of SD loaded Ag-MCM-41, Ag-SBA-15 materials and AgSD loaded MCM-41 and SBA-15 samples

\begin{tabular}{|c|c|c|c|c|c|c|c|c|}
\hline Bacterial strains & \multicolumn{8}{|c|}{ Zone of inhibition $[\mathrm{mm}]$} \\
\hline Escherichia coli ATCC 8739 & $1+7$ & $1+6$ & $1+7$ & $1+7$ & $1+7$ & $1+10$ & $1.5+6.5$ & $2+5$ \\
\hline Staphylococcus aureus ATCC 6538 & 1.5 & 1.5 & 1 & 1 & 1 & 1 & 2 & 2 \\
\hline
\end{tabular}

spectrum of AgSD loaded into the silica carrier. The particular spectral pattern in the region between 156 and $161 \mathrm{ppm}$ could be explained by the distribution of AgSD molecules within the pores and on the outer surface.

Fig. 5C and D show the ${ }^{13} \mathrm{C}$ NMR spectra of pure SD and SD adsorbed on silver-modified SBA-15 material (Ag-SBA-15). Comparing these spectra, it can be observed that all ${ }^{13} \mathrm{C}$ signals are shifted downfield by an average of $0.32 \mathrm{ppm}$ in SD/Ag-MCM41 material. This result indicates that SD molecules in silvermodified SBA-15 material are predominantly adsorbed in the pores. ${ }^{13} \mathrm{C}$ NMR investigations are in a good agreement with the XRD and FT-IR data.

The amount of sulfadiazine loaded in the mesopores of MCM-41 and SBA-15 and their Ag-modified analogues was quantified using thermogravimetry (TG). The Ag-MCM-41 sample showed lower adsorption capacity (30.5 wt\%) for SD in comparison to its AgSBA-15 analogue (45.8 wt\%) (Fig. 6A).
MCM-41 is composed of $100 \mathrm{~nm}$ spherical particles, and among the particles secondary mesopores can be found. Therefore, higher SD adsorption capacity should be expected for Ag-MCM41. Moreover, nitrogen physisorption experiments show high pore-filling values. Thus, the lower SD adsorption capacity can be explained only by the blocking of pore entrances due to the narrower pores of Ag-MCM- 41 host. Interaction of SD with silver ions on the outer surface of MCM-41 can also contribute to this pore blocking effect. The loading of AgSD on MCM-41 and SBA15 silicas resulted in $26.0 \mathrm{wt} \%$ and $30.7 \mathrm{wt} \%$ adsorbed sulfadiazine, respectively (Fig. 6B). Taking into account that in contrast to SD only $70 \%$ of AgSD can be decomposed by thermal treatment up to $600{ }^{\circ} \mathrm{C}$ (metallic silver remains on the surface after decomposition of organic material), the above values correspond to $78 \%$ and $92 \%$ of the loaded drug. The AgSD content is higher on MCM-41 compared to SD loaded on AgMCM-41, and similar to SBA-15 and Ag-SBA-15 carriers. These

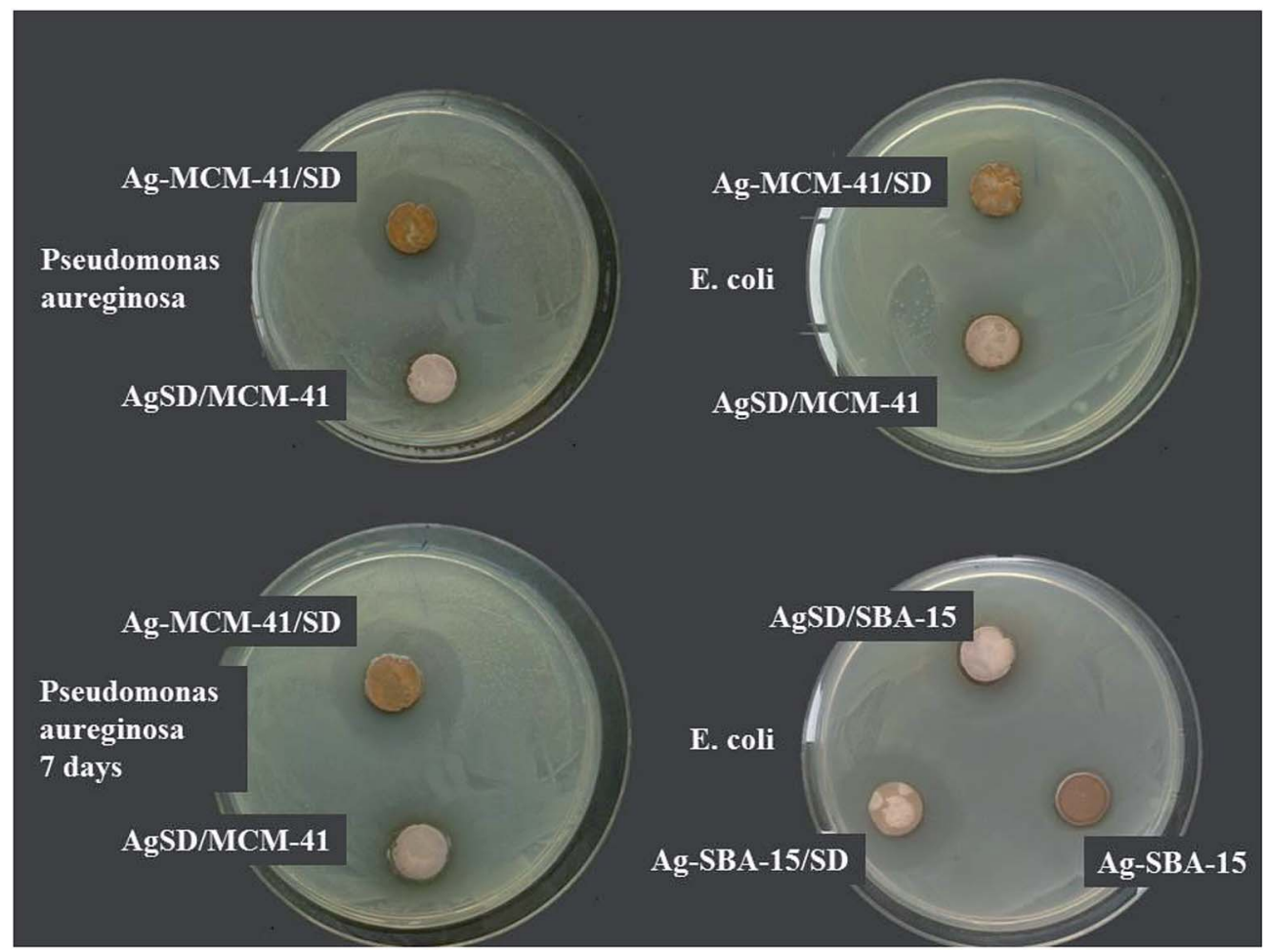

Fig. 8 Photos of agar plates inoculated with Escherichia coli (ATCC 8739) and Pseudomonas aeruginosa (ATCC 9027) illustrating the inhibition zones for Ag-MCM-41/SD, AgSD/MCM-41, Ag-SBA-15/SD and AgSD/SBA-15 samples investigated by the pressed discs method. 
results show that similarly efficient SD loading can be achieved by the application of Ag-functionalized silica carriers.

\subsection{In vitro release of SD and AgSD}

The in vitro release of silver sulfadiazine from MCM-41 and SBA15 samples and sulfadiazine from silver-modified ones was studied in a phosphate buffer $(\mathrm{pH}=5.5)$. The latter $\mathrm{pH}$ value is widely applied for in vitro experiments for dermatological formulations. The drug release profiles are presented in Fig. 7. As seen in Fig. 7, non-encapsulated AgSD is poorly dissolved in the selected medium, which could be expected considering its poor water solubility.

In contrast, the loading of AgSD on mesoporous carriers resulted in improved dissolution rate (Fig. 7A). This can be explained by the lower crystallinity of the loaded AgSD, i.e. a part of it can be in nanocrystalline or in amorphous form. The formation of nanocrystalline or amorphous particles is predetermined by the adsorption of AgSD on the high surface of mesoporous silica and by the penetration into the pore system. Comparing the different nanoporous carriers, faster release of AgSD was registered from SBA-15 particles, which can be explained by the larger size of the pores. Note that improvement of AgSD dissolution rate is advantageous because the better solubility of the drug can enhance its antibacterial activity.

Similarly, the release of sulfadiazine from Ag-modified SBA15 carrier was faster compared to MCM-41 one (Fig. 7B). However, non-loaded sulfadiazine showed similar release profile to that of loaded into Ag-SBA-15 carrier. Adsorption of sulfadiazine on Ag-modified MCM-41 carrier resulted in a prolonged release. The improved solubility of AgSD and the reduced burst release of both SD and AgSD on MCM-41 can be advantageous in the development of topical drug delivery system for wound treatment.

The stability of the drug delivery system was checked by exposing the samples to direct daylight for 5 days. Repeated drug delivery experiments did not show significant differences between the parent and irradiated samples.

\subsection{Antibacterial efficacy of SD loaded Ag-MCM-41 and Ag- SBA-15 samples}

Our silver-containing samples were tested against the most commonly found burn wound bacteria ${ }^{40}$ such as strains of ATCC Escherichia coli 8739, Staphylococcus aureus 6538, Pseudomonas aeruginosa 9027, and Streptococcus pyogenes 19615. Results of antibacterial investigations are summarized in Tables 2 and 3, and some example photos of agar plates are shown in Fig. 8. Silver-containing nanoporous silica materials and SD itself have some inherent antibacterial properties. Therefore, these samples were investigated as control ones. In most cases, investigating Escherichia coli and Pseudomonas aeruginosa strains, two zones appeared inside the cultures, i.e. a double ring could be observed. The inner zone was clear, where the bacteria could not grow at all (1-2 mm wide), whereas the exterior, wider zone was less translucent (5-10 mm). Here, the growth of bacteria was inhibited compared to the parent population.
The silver-containing nanoporous silica control samples demonstrated identical or smaller inhibition zones than the SDloaded varieties. SD-loaded pure silica MCM-41 showed no antimicrobial effect, except for E. coli. However, in this case, there was no totally clear inhibition zone, only a less translucent one. Ag-SBA-15 based samples exhibited smaller inhibition zones than Ag-MCM-41 ones, probably in correlation with the higher silver dissolution rate of the latter one $\left(4.7 \mathrm{mg} \mathrm{g}^{-1}\right.$ for AgMCM-41 compared to $2.9 \mathrm{mg} \mathrm{g}^{-1}$ for Ag-SBA-15). SD-loaded AgMCM-41 and Ag-SBA-15 samples showed similar inhibition zones, in some cases larger ones than AgSD-loaded pure silica ones. According to the photos of agar plates (not shown), when the diameter of inhibition zones was identical the outer, less transparent zone was much clearer for the Ag-MCM41/SBA-15/ SD samples compared to AgSD-loaded MCM-41 and SBA-15 ones. These results indicate that SD-loaded silver nanoporous silica samples are equally or even better effective in bacterial inhibition than AgSD.

Moreover, our experiments supported the well-known fact ${ }^{\mathbf{4 1}}$ that ionic silver, a heavy metal with relatively broad antibacterial spectrum, has stronger effect on antibacterial activity than pure SD. The beneficial effect of silver is so strong that agar plates showed the original inhibition zones even after 7 days of incubation (Fig. 8).

\section{Conclusions}

In this study, it was shown that silver modified MCM-41 and SBA-15 materials are suitable carriers for drugs such as sulfadiazine to design a drug-delivery system with improved bioavailability and antibacterial activity. It was found that nanoporous silica materials can be easily modified by direct or post-synthesis methods to prepare silver nanoparticles inside the channels or on the outer surface of the particles. By these methods, the amount of incorporated silver can be controlled in a relatively wide concentration range between 0 and $10 \mathrm{wt} \%$. The silica host can stabilize these silver nanoparticles and water-soluble silver ions are released. The empty channels of the silica support are suitable for the storage of relatively high amount of drug molecules. We demonstrated for the first time that, comparing the SD release and antimicrobial properties, AgSD can be effectively replaced by SD-loaded Ag-MCM-41 or AgSBA-15 materials. Adsorbing AgSD on pure nanoporous silica materials significantly improved its water solubility.

\section{Acknowledgements}

Financial support by the Bulgarian-Hungarian Inter-Academic Exchange Agreement is greatly acknowledged.

\section{Notes and references}

1 Z. Aziz, S. F. Abu and N. J. Chong, Burns, 2012, 38, 307.

2 B. S. Atiyeh, M. Costagliola, S. N. Hayek and S. A. Dibo, Burns, 2007, 33, 139.

3 D. S. Cook and M. F. Turner, J. Chem. Soc., Perkin Trans. 2, 1975, 1021. 
4 N. C. Baenziger and A. W. Struss, Inorg. Chem., 1976, 15, 1807. 5 A. Bult and H. B. Klasen, Arch. Pharm., 1980, 313, 1016.

6 C. L. Fox, Int. Surg., 1975, 60, 275.

7 C. L. Fox and S. M. Modak, Antimicrob. Agents Chemother., 1974, 5, 582.

8 J. Nesamony and W. M. Kolling, J. Pharm. Sci., 2005, 94, 1310. 9 M. Venkataraman and M. Nagarsenker, AAPS PharmSciTech, 2013, 14, 254.

10 L. Catenacci, M. Sorrenti, G. Bruni, M. C. Bonferoni, G. Sandri and G. Bettinetti, J. Therm. Anal. Calorim., 2013, 111, 2149.

11 S. J. Strydom, W. E. Rose, D. P. Otto, W. Liebenberg and M. M. de Villiers, Nanomedicine: Nanotechnology, Biology, and Medicine, 2013, 9, 85.

12 A. Lichtenstein and R. Margalit, J. Inorg. Biochem., 1995, 60, 187.

13 A. Delrivo, A. Zoppi and M. Raquel Longhi, Carbohydr. Polym., 2012, 87, 1980.

14 Z. B. Cao, X. B. Sun, Y. Y. Sun and H. Fong, J. Bioact. Compat. Polym., 2009, 24, 350.

15 A. R. Fajardo, L. C. Lopes, A. O. Caleare, E. A. Britta, C. V. Nakamura, A. F. Rubira and E. C. Muniz, Mater. Sci. Eng., C, 2013, 33, 588.

16 D. Mangindaan, C.-T. Chen and M.-J. Wang, Appl. Surf. Sci., 2012, 262, 114.

17 Y.-S. Cho, J.-W. Lee, J.-S. Lee, J.-H. Lee, T. R. Yoon, Y. Kuroyanagi, M. H. Park, D. G. Pyun and H. J. Kim, J. Mater. Sci.: Mater. Med., 2002, 13, 861.

18 H.-J. Kim, E.-Y. Choi, J.-S. Oh, H.-C. Lee, S.-S. Park and C.-S. Cho, Biomaterials, 2000, 21, 131.

19 F.-L. Mi, Y.-B. Wu, S.-S. Shyu, A.-C. Chao, J.-Y. Lai and C.-C. Su, J. Membr. Sci., 2003, 212, 237.

20 Y. M. Lee, S. S. Kim, M. H. Park, K. W. Song, Y. K. Sung and I. K. Kang, J. Mater. Sci.: Mater. Med., 2000, 11, 817.

21 M. Vallet-Regi, A. Ramila, R. P. del Real and J. Perez-Pariente, Chem. Mater., 2001, 13, 308.

22 S. Wang, Microporous Mesoporous Mater., 2009, 117, 1.

23 A. Szegedi, M. Popova, I. Goshev, S. Klébert and J. Mihály, J. Solid State Chem., 2012, 194, 257.
24 M. D. Popova, Á. Szegedi, I. N. Kolev, J. Mihály, B. S. Tzankov, G. T. Momekov, N. G. Lambov and K. P. Yoncheva, Int. J. Pharm., 2012, 436, 778.

25 W. Zhu, Y. Han and L. An, Microporous Mesoporous Mater., 2005, 80, 221.

26 W. Gac, A. Derylo-Marczewska, S. Pasieczna-Patkowska, N. Popivnyak and G. Zukocinski, J. Mol. Catal. A: Chem., 2007, 268, 15.

27 S. Huh, J. Wiench, J.-C. Yoo, M. Pruski and V. S.-Y. Lin, Chem. Mater., 2003, 15, 4247.

28 M. Iwamoto and Y. Tanaka, Catal. Surv. Jpn., 2001, 5, 25.

29 D. Zhao, J. Feng, Q. Huo, N. Melosh, G. H. Fredrickson, B. F. Chmelka and G. D. Stucky, Science, 1998, 279, 548.

30 H. Kunkely and A. Vogler, Inorg. Chem. Commun., 2007, 10, 226.

31 B. D. Cullity, Elements of X-ray Diffraction, Addison-Wesley, Reading, Mass, USA, 1978.

32 O. Prymak, S. Ristig, V. Meyer-Zaika, A. Rostek, L. Ruiz, J. M. González-Calbet, M. Vallet-Regi and M. Epple, Russ. Phys. J., 2014, 56, 1111.

33 Á. Szegedi, Z. Kónya, D. Méhn, E. Solymár, G. Pál-Borbély, Z. E. Horváth, L. P. Biró and I. Kiricsi, Appl. Catal., A, 2004, 272, 257.

34 M. Rai, A. Yadav and A. Gade, Biotechnol. Adv., 2009, 27, 76. 35 A. Galarneau, M. Nader, F. Guenneau, F. Di Renzo and A. Gedeon, J. Phys. Chem. C, 2009, 111, 8268.

36 M. Kawashita, S. Tsuneyama, F. Miyaji, T. Kokubo, H. Kozuka and K. Yamamoto, Biomaterials, 2000, 21, 393.

37 R. Lu, W. Zou, H. Du, J. Wang and S. Zhang, Ceram. Int., 2014, 40, 3693.

38 G. Ogruc-Ildiz, S. Akyuz and A. E. Ozle, J. Mol. Struct., 2009, 924-926, 514.

39 H. Kunkey and A. Vogler, Inorg. Chem. Commun., 2007, 10, 226.

40 D. Church, S. Elsayed, O. Reid, B. Winston and R. Lindsay, Clin. Microbiol. Rev., 2006, 19, 403.

41 M. K. Rai, S. D. Deshmukh, A. P. Ingle and A. K. Gade, J. Appl. Microbiol., 2012, 112, 841. 